\title{
Study of FCNC in top quark production and decay with the ATLAS detector
}

\author{
J.P. Araque, on behalf of the ATLAS Collaboration* \\ $\dagger$ \\ Laboratório de Instrumentação e Física Experimental de Partículas, Departamento de Física da \\ Universidade do Minho, Campus de Gualtar, 4710-057 Braga, Portugal \\ E-mail: jp.araque@cern.ch
}

\begin{abstract}
A review of the analyses involving flavour changing neutral currents (FCNC) processes using proton-proton collisions produced at the LHC and data collected with the ATLAS detector is presented. Results of the analyses searching for top-quark decay through $Z$ and Higgs boson, as well as direct top-quark production are considered. A quick overview on the expected sensitivity for the high-luminosity LHC (HL-LHC) and results of other analyses searching for new models in which FCNC processes are allowed, are presented.
\end{abstract}

The 15th International Conference on B-Physics at Frontier Machines at the University of Edinburgh 14 -18 July, 2014

University of Edinburgh, $U K$

\footnotetext{
* Speaker.

$\dagger$ J.P. Araque is funded by the FCT grant SFRH/BD/52002/2012 QREN, FCOMP-01-0124-FEDER-022818 CERN/FP/123595/2011
} 


\section{Introduction}

The Standard Model (SM) offers the best description of the subatomic world known so far. Nonetheless, the SM does not explain the fermion generations and mass hierarchy, nor explain the asymmetry of matter-antimatter in the universe. In that sense, the SM cannot be considered as a complete theory and new models appear trying to address these open questions. These new models usually imply either the introduction of new particles or new interactions between the existing ones. A common feature of several beyond SM (BSM) models is the presence of flavour changing neutral currents. In the SM, this is forbidden by the GIM mechanism at tree level and highly suppressed at higher perturbative orders. In these new models the FCNC branching ratios can be enhanced by several orders of magnitude, providing an optimal scenario to search for new physics beyond the SM.

In the following sections, several ATLAS [1] analyses are presented targeting different FCNC processes.

\section{FCNC processes in top-quark decay: $t \rightarrow Z q$}

One possible scenario is the decay of a top-quark through a $Z$ boson $(t \rightarrow Z q, q=u, c)$ [2]. This analysis was done with an integrated luminosity of $2.1 \mathrm{fb}^{-1}$ at $\sqrt{s}=7 \mathrm{TeV}$ collected with the ATLAS detector. It focuses on the FCNC decay of the top-quark in $t \bar{t}$ events with one of the topquarks decaying through a $Z$ boson via an FCNC process and the other top-quark through charged currents $(t \rightarrow W b)$. Events with three leptons (only $e$ and $\mu$ are considered) were selected and two different channels were defined based on the information used to reconstruct them:

- The three leptons in the event are reconstructed using both tracking and calorimeter information. This is noted as the 3ID channel.

- Two of the leptons are ID leptons, reconstructed with tracking and calorimeter information, and the third lepton is reconstructed using only tracking information. This is done in order to increase signal sensitivity and is noted as the $2 \mathrm{ID}+1 \mathrm{TL}$ channel.

In both channels, at least a pair of same-flavour opposite-sign (SF-OS) leptons must exist to reconstruct the $Z$ boson. The presence of at least 2 jets was also required and, in the 2ID+1TL channel, an additional requirement of at least one $b$-jet was imposed to minimise the contribution from backgrounds with fake leptons. The missing transverse energy $\left(E_{\mathrm{T}}^{\text {miss }}\right)$ was required to be grater than $20 \mathrm{GeV}$ and the invariant mass of the two SF-OS leptons was required to be within a $15 \mathrm{GeV}$ windows around the $Z$ mass peak.

The $t \bar{t}$ system was reconstructed using a $\chi^{2}$ minimisation in which the selected events were required to be consistent with the $t \bar{t} \rightarrow Z q W b$ topology. All possible combinations of jets and leptons were taken into account, requiring that the $Z$ boson candidate is built with SF-OS leptons. The longitudinal component of the neutrino momentum $p_{Z}^{v}$ was also obtained from the minimisation. The $\chi^{2}$ was defined as:

$$
\chi^{2}=\frac{\left(m_{j_{a} l_{a} l_{b}}^{\text {reco }}-m_{t}\right)^{2}}{\sigma_{t}^{2}}+\frac{\left(m_{j_{b} l_{c} v}^{\text {reco }}-m_{t}\right)^{2}}{\sigma_{t}^{2}}+\frac{\left(m_{l_{c} v}^{\text {reco }}-m_{W}\right)^{2}}{\sigma_{W}^{2}}+\frac{\left(m_{l_{a} l_{b}}^{\text {reco }}-m_{Z}\right)^{2}}{\sigma_{Z}^{2}},
$$


where $j_{a, b}$ are the two highest transverse momentum $\left(p_{\mathrm{T}}\right)$ jets and $l_{a, b, c}$ are the three lepton candidates. The constraints were defined as follows: $m_{t}=172.5 \mathrm{GeV}, m_{W}=80,4 \mathrm{GeV}$ and $m_{Z}=$ $91.2 \mathrm{GeV}$. The widths were derived from MC studies and set to $\sigma_{t}=14 \mathrm{GeV}, \sigma_{W}=10 \mathrm{GeV}$ and $\sigma_{Z}=3 \mathrm{GeV}$.

The main backgrounds for this analysis come from processes with 3 real leptons (diboson ZZ and WZ), evaluated using Monte Carlo simulation, events with 2 real leptons and 1 fake lepton, evaluated via data-driven methods using the $Z$ peak as control region divided in different $E_{\mathrm{T}}^{\text {miss }}$ bins and events with fake track lepton, derived using a $\gamma+$ jets data sample selected with photon triggers. Diboson processes were the dominant background in the 3ID channel as shown in Figure 1(a) while events with fake track leptons dominated the 2ID+1TL channel as shown in Figure 1(b). The dominant systematic uncertainties were diboson background modelling, jet energy scale and $t \bar{t}$ normalisation.

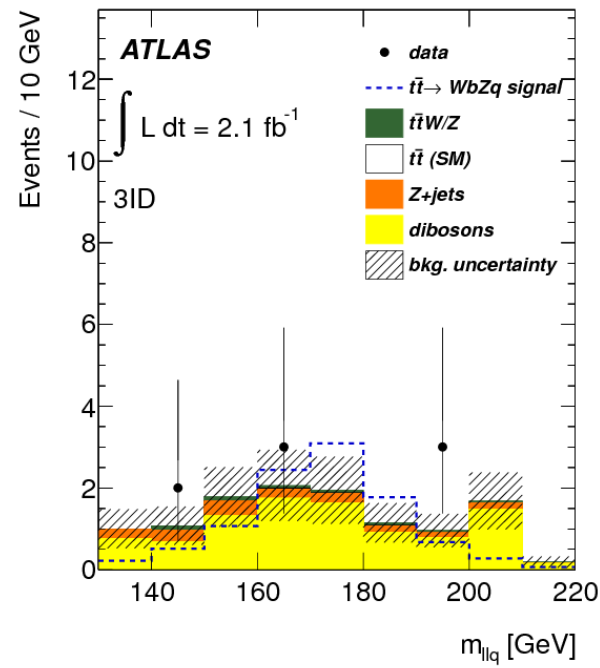

(a)

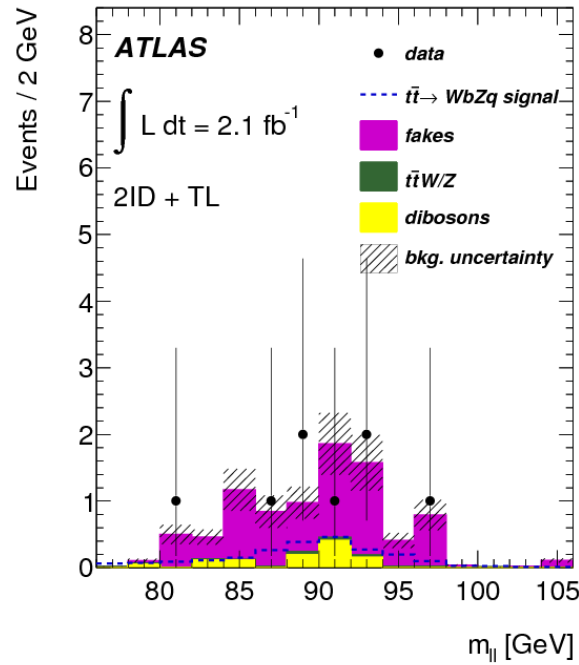

(b)

Figure 1: Distributions after selections for (a) the invariant mass of the FCNC decaying top-quark in the 3ID channel and (b) the invariant mass of the $Z$ boson in the 2ID+1TL channel [2].

In the absence of an evidence for the searched signal, the $C L_{s}$ method [3] was used to set upper limits in the branching ratios for the FCNC process. Both 3ID and 2ID+1TL channel are combined and an observed (expected) upper limit of $0.73 \%(0.93 \%)$ is found at a $95 \%$ confidence level (C.L.).

\section{FCNC in top-quark decay: $t \rightarrow H q$ with $H \rightarrow \gamma \gamma$}

This analysis was performed using data collected with the ATLAS detector corresponding to $4.1 \mathrm{fb}^{-1}$ at $\sqrt{s}=7 \mathrm{TeV}$ and $20.3 \mathrm{fb}^{-1}$ at $\sqrt{s}=8 \mathrm{TeV}$ [4]. It targets the FCNC decay of the top-quark through a Higgs boson and a lighter, same-charge quark in $t \bar{t}$ events in which the other top-quark decays through charged currents $(t \rightarrow W b)$. 
Events were selected with at least two photons $\left(p_{\mathrm{T}}\left(\gamma_{1}\right)>40 \mathrm{GeV}\right.$ and $\left.p_{\mathrm{T}}\left(\gamma_{2}\right)>30 \mathrm{GeV}\right)$ and further selection was done depending on whether the $W$ boson from the SM decaying top-quark has an hadronic or leptonic decay:

- Hadronic channel: events were selected with no leptons and 4 jets, with at least one of them being a $b$-tagged jet. The reconstructed top masses were required to lie within certain mass windows around the top quark mass. In the case of the FCNC decaying top-quark the window is defined as $[156,192] \mathrm{GeV}$ and for the SM decaying top-quark $[130,210] \mathrm{GeV}$.

- Leptonic channel: the analysis in the leptonic channel was only performed with $8 \mathrm{TeV}$ data. Events were required to have exactly one lepton and at least 2 jets with exactly one of them being a $b$-tagged jet. The reconstruction of the FCNC decaying top-quark follows the same criteria adopted in the hadronic channel while the SM decaying top-quark mass is required to be inside the $[135,205] \mathrm{GeV}$ window.

The background contribution was estimated from a sideband fit to data of the invariant mass of the two photons $\left(m_{\gamma \gamma}\right)$ distribution with the dominant contribution being $\gamma \gamma j$ as can be seen in Figure 2(a). The control regions for the sideband fit were defined as $100 \mathrm{GeV} \geq m_{\gamma \gamma} \geq 122 \mathrm{GeV}$ and $129 \mathrm{GeV} \geq m_{\gamma \gamma} \geq 160 \mathrm{GeV}$ while the signal region is defined as $122 \mathrm{GeV} \geq m_{\gamma \gamma} \geq 129 \mathrm{GeV}$.

Since no evidence of new physics was found, the $C L_{s}$ method was used to set upper limits on the branching ratios for the FCNC decay. The $m_{\gamma \gamma}$ distribution was used as discriminant variable in the hadronic selection and a counting experiment was performed in the leptonic selection. The observed (expected) upper limit on the branching ratio was found to be $0.79 \%(0.51 \%)$ at a $95 \%$ C.L. as shown in Figure 2(b).

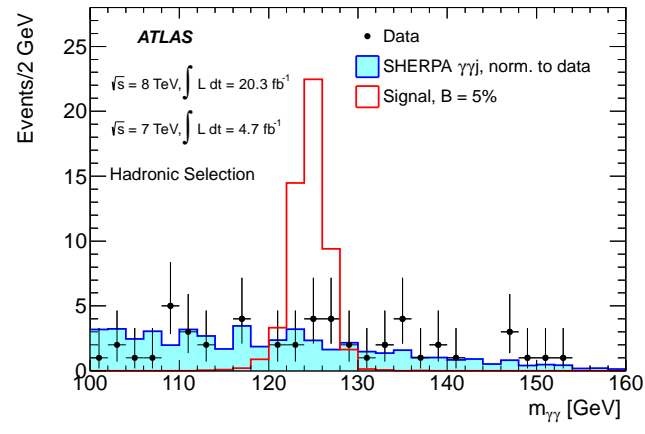

(a)

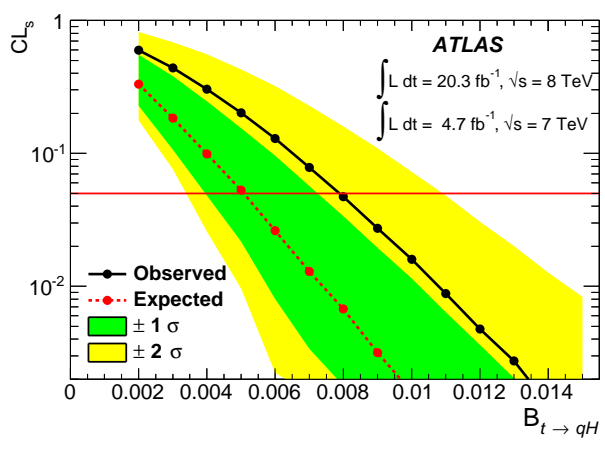

(b)

Figure 2: The final discriminant variable (a) $m_{\gamma \gamma}$ is shown for hadronic selection. The $C L_{s}$ value (b) is shown as a function of the branching ratios for the top-quark decay to a Higgs boson [4].

\section{FCNC in direct top-quark production}

Among the FCNC processes involving the top-quark, it is possible to have the top-quark coupled with an up or a charm quark and a gluon. However, a process like $t \rightarrow g q$ is almost indistinguishable from the overwhelming multijet background. Nonetheless, this coupling can be studied 
through direct top-quark production, $g q \rightarrow t$. This analysis was done with $14.2 \mathrm{fb}^{-1}$ of data collected with the ATLAS detector at $\sqrt{s}=8 \mathrm{TeV}$ [5]. Two main differences can be found when comparing direct top-quark production with SM single-top production. First, in direct top-quark production the transverse momentum of the top-quark arises from initial state QCD radiation, with the resulting $W$ and $b$ decay products of the top-quark tending to be back to back in contrast to SM single-top production. Second, the FCNC process is expected to produce three times more top-quarks than top anti-quarks when in the SM single-top production this ratio is at most two.

In this analysis the top-quark decays through the SM $W b$ decay and events were selected being compatible with this assumption: exactly one lepton with $p_{\mathrm{T}}>25 \mathrm{GeV}, E_{\mathrm{T}}^{\mathrm{miss}}>30 \mathrm{GeV}$ and exactly one $b$-tagged jet.

The main background processes were estimated to be single-top production, $t \bar{t}$ and $W / Z+$ jets. The multijet background was estimated separately for the electron and muon channel. For the electron channel the shape was derived from a Sherpa di-jet sample while the normalisation was obtained by fitting the $E_{\mathrm{T}}^{\text {miss }}$ distribution to data in the central and forward region. In the muon channel a matrix-method was used to obtain both shape and normalisation. The systematic uncertainties were dominated by $b$-tagging, $E_{\mathrm{T}}^{\text {miss }}$ and background modelling.

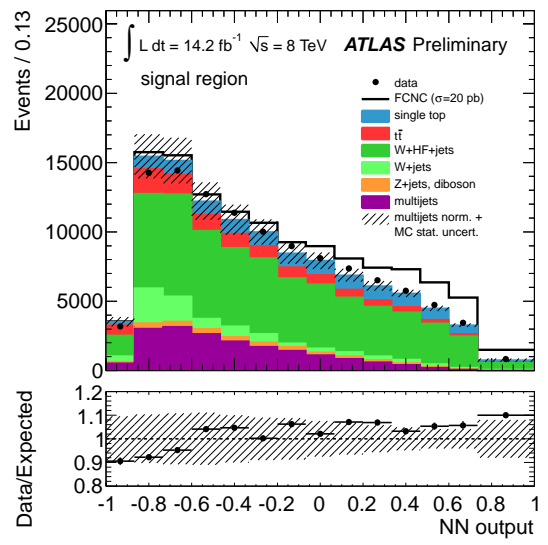

(a)

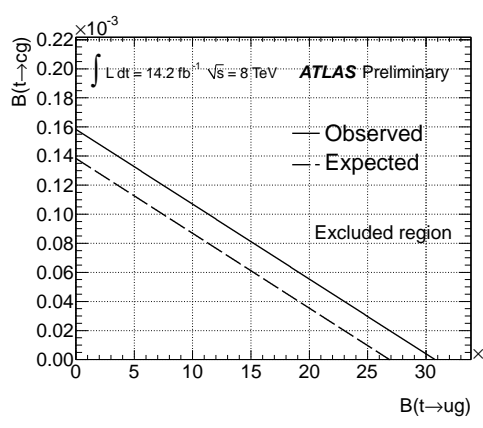

(b)

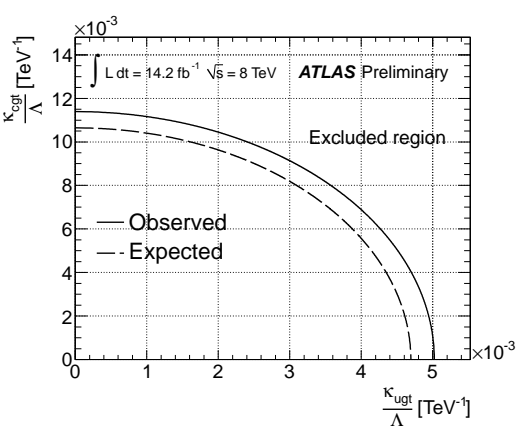

(c)

Figure 3: Direct top-quark production distributions are shown for (a) the output of the neural network, (b) expected and observed exclusion region and a 95\% C.L. as a function of the branching ratios of the top-quark decaying to a gluon and a $u$ and $c$ quark, (c) expected and observed exclusion regions at a 95\% C.L. as a function of the couplings, derived assuming $B R(t \rightarrow W b)=1$ and using the next-to-leading-order theoretical cross-section prediction [5].

In this analysis there was no single observable with enough discriminant power to differentiate between signal and background, so a multi-variate analysis was used. The main observables driving most of this analysis sensitivity were found to be the transverse momentum of the $b$-quark, the transverse momentum of the $W$ boson and the difference in the azimuthal angle between the $W$ boson and the neutrino, derived in the top-quark rest frame. The output of the neural network is shown in Figure 3(a).

Since no significant deviation from the SM is found, upper limits on both the branching ratio 
of $t \rightarrow g q$ and the FCNC couplings were derived. The exclusion regions are shown in Figures 3(b) and (c).

\section{Search for new vector-like quarks decaying through FCNC}

FCNC processes can be found in many different models involving top-quarks and the ATLAS Collaboration performed several searches which are relevant in this context. One example is the search for vector-like quarks (VLQ), which are predicted in several models beyond the SM. VLQ are heavy quarks whose left and right chiralities transform in the same way under the $S U(2)$ group of the $\mathrm{SM}$ and can be found with electric charges of $-2 / 3,-1 / 3,2 / 3$ and $5 / 3$ for the vector-like $Y, B, T$ and $X$ respectively. FCNC processes can be found in VLQ decays through $Z$ and Higgs boson, providing a new channel for top-quark production through vector-like $T$ decay $(T \rightarrow Z / H t)$.

Several VLQ searches have been performed in ATLAS targeting different decay channels [6, $7,8,9]$. Figure 4 shows the lower limits on the VLQ masses as a function of the branching ratios to $W$ and Higgs boson superimposing all analyses results for vector-like $B$ and vector-like $T$. As can be seen, masses below $\sim 650 \mathrm{GeV}$ have been excluded for all branching ratios.
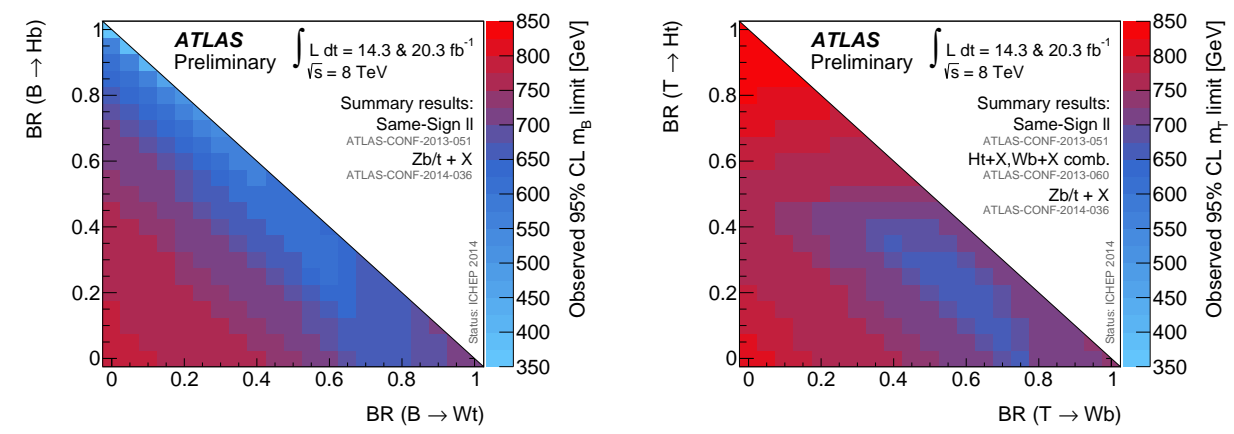

Figure 4: Observed maximum excluded mass for vector-like $B$ and for vector-like $T$ [10].

\section{Future prospects}

In the LHC second operation phase and further in the future (HL-LHC), the centre of mass energy will increase up to $14 \mathrm{GeV}$ and the integrated luminosity is expected to reach up to $3 \mathrm{ab}^{-1}$. This will allow new models to be tested with an unprecedented precision.

Most recent results in FCNC top-quark decay through a $Z$ boson exclude branching ratios above $\sim 5 \cdot 10^{-4}[11]$, and in the case of the $t \rightarrow q \gamma$ channel, branching ratios above $2 \cdot 10^{-4}\left(10^{-3}\right)$ are excluded where $q=u(c)$ [12]. As can be seen in Figure 5(a), these results can be improved when extrapolated to the HL-LHC conditions, being able to achieve sensitivity of branching ratios of the order $\sim 10^{-5}[13]$.

In the case of the FCNC decay of the top-quark through a Higgs boson a significant increase in sensitivity is also expected, achieving sensitivity to branching ratios of the order of $\sim 10^{-4}$, as can be seen in Figure 5(b) [14]. 


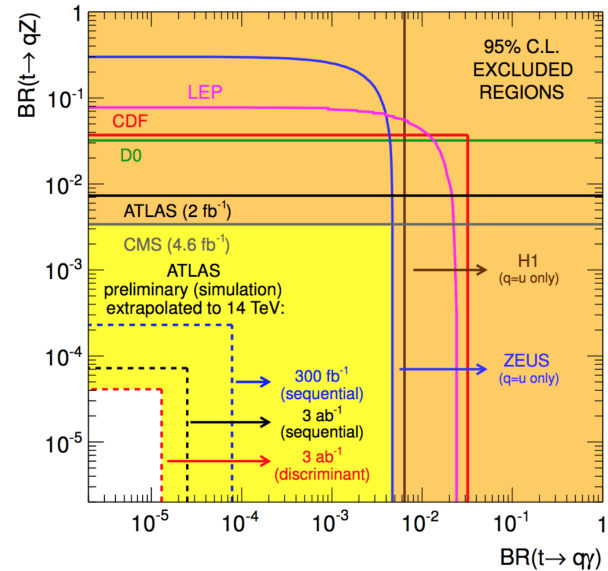

(a)

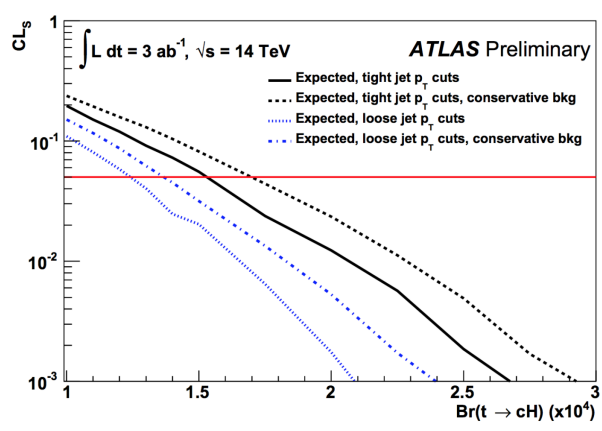

(b)

Figure 5: Future prospects for FCNC upper limits for (a) branching ratios of top-quarks to a $Z$ boson and a photon [13] and (b) top-quark to a Higgs boson [14] in the high luminosity LHC era.

\section{References}

[1] ATLAS Collaboration, JINST 3 S08003 (2008).

[2] ATLAS Collaboration, JHEP 1209, 139 (2012).

[3] A. L. Read, J. Phys. G 28, 2693 (2002).

[4] ATLAS Collaboration, JHEP 1406, 008 (2014).

[5] ATLAS Collaboration, ATLAS-CONF-2013-063, http://cds.cern.ch/record/1562777.

[6] ATLAS Collaboration, ATLAS-CONF-2013-051, http://cdsweb.cern.ch/record/1547567.

[7] ATLAS Collaboration, ATLAS-CONF-2013-060, http://cdsweb.cern.ch/record/1557777.

[8] ATLAS Collaboration, ATLAS-CONF-2013-018, http://cdsweb.cern.ch/record/1525525.

[9] ATLAS Collaboration, arXiv:1409.5500 [hep-ex].

[10] ATLAS Collaboration, http://atlas.web.cern.ch/Atlas/GROUPS/PHYSICS/CombinedSummaryPlots/EXOTICS/.

[11] CMS Collaboration, Phys. Rev. Lett. 112, 171802 (2014).

[12] CMS Collaboration, CMS-PAS-TOP-14-003, http://cds.cern.ch/record/1700519.

[13] ATLAS Collaboration, ATL-PHYS-PUB-2012-001, http://cds.cern.ch/record/1472518.

[14] ATLAS Collaboration, ATL-PHYS-PUB-2013-012, http://cds.cern.ch/record/1604506. 